\title{
Thoracic Spinal Cord Stab Injury: A Case Report and Literature Review
}

\author{
Aurélien Ndoumbe ${ }^{1 *}$, Marc Leroy Guifo², Mathieu Motah ${ }^{3}$, Samuel Takongmo ${ }^{2}$ \\ ${ }^{1}$ Faculty of Medicine \& Pharmaceutical Sciences, University of Douala, Douala, Cameroon \\ ${ }^{2}$ Faculty of Medicine \& Biomedical Sciences, University of Yaoundé I, CHU of Yaoundé, Yaoundé, Cameroon \\ ${ }^{3}$ Faculty of Medicine \& Pharmaceutical Sciences, University of Douala, Douala General Hospital, Douala, \\ Cameroon \\ Email: ${ }^{*}$ aurelien.ndoumbe@gmail.com
}

Received 20 August 2015; accepted 9 October 2015; published 12 October 2015

Copyright (C) 2015 by authors and Scientific Research Publishing Inc.

This work is licensed under the Creative Commons Attribution International License (CC BY).

http://creativecommons.org/licenses/by/4.0/

$$
\text { (c) (i) Open Access }
$$

\section{Abstract}

Thoracic spinal cord stab injuries are rare lesions. A 17-year-old boy was stabbed on his back by his classmate when he bent forward to pick up his cloth from the ground. On admission, he presented with: complete paraplegia with muscle strength of zero on all muscle groups, complete anesthesia from dermatome $\mathbf{1 0}$ and below, acute urinary retention, and a four-centimeter wound on the thoracolumbar region from which cerebrospinal fluid mixed with blood was oozing out. A high-dose methylprednisolone protocol was started $(30 \mathrm{mg} / \mathrm{kg}$ in one hour and then $5.4 \mathrm{mg} / \mathrm{kg}$ over 23 hours) an indwelling urinary catheter placed and sterile dressing of the wound done. Antibiotics and analgesics were also administered. The computed tomography scanning revealed a spinal cord transection at T10 - T11 level with incarceration of the broken knife blade. An emergency thoracic laminectomy was performed. Removal of the broken knife blade revealed complete spinal cord transection with a compressive hematoma within the spinal cord which was removed by smooth suction. The spinal dura was sutured and the wound closed in many layers. On day 14 after surgery, sensitivity was recovered with 3 on 5 muscle strength in both lower limbs except for both feet where motor function remained null. Urinary retention and fecal incontinence persisted. The patient was discharged from our service for a rehabilitation center. At 32-month follow-up, neurological examination was unchanged although patient noticed a slight improvement of sphincter disturbances.

\section{Keywords}

Spinal Cord Injury, Thoracic Spine, Stab Wound, Knife, Seventeen-Year-Old Boy

\footnotetext{
${ }^{*}$ Corresponding author.
}

How to cite this paper: Ndoumbe, A., Guifo, M.L., Motah, M. and Takongmo, S. (2015) Thoracic Spinal Cord Stab Injury: A Case Report and Literature Review. Open Journal of Modern Neurosurgery, 5, 113-117. 


\section{Introduction}

Spinal cord stab injuries (SCSI) are rare traumatic lesions when compared to injuries caused by road traffic accidents, sport activities and guns [1]-[5]. The disability resulting from spinal cord injuries (SCI) puts a heavy burden on victims and the whole society concerning quality of life, working time loss and financial cost [6]. The initial management of SCI is crucial for protecting undamaged spinal cord from secondary insults. In spite of scientific progresses, it is still not possible yet to repair a damaged spinal cord. Authors are reporting on a case of thoracic spinal cord stab injury in a 17-year-old boy, with incarceration of a broken knife blade. The patient was stabbed on his back by his classmate. He presented with complete paraplegia and sensory loss at thoracic level 10.

\section{Clinical Case}

A 17-year-old boy was admitted to the emergency department of our hospital after he was stabbed on the back by his classmate while he bent forward to pick up his clothes from the ground. The broken knife blade remained incarcerated in the wound. On admission, the patient was unable to move his lower limbs, he was complaining of back pain and cerebrospinal fluid (CSF) mixed with blood was leaking from the wound. Physical examination revealed: a 4 centimeter parasagittal wound located at the thoracolumbar area on the right side (Figure 2), there was complete flaccid paraplegia with muscle strength of zero on all muscle groups, abolished deep tendon reflexes on lower limbs, indifferent plantar reflexes, complete anesthesia from level T10 and below, urinary retention, and hypotonic anal sphincter with abolished bulbo-cavernosus reflex.

In the emergency Ward, sterile dressing of the wound was done, an intravenous line was inserted, blood samples were collected, and antibiotics were started along with high dose methylprednisolone (Houde laboratories, Paris, France) protocol (30 mg/kg IV infusion in one hour and then $5.4 \mathrm{mg} / \mathrm{kg}$ given over 23 hours). After that, a computed tomography (CT, Toshiba, Tokyo, Japan) scan of thoracolumbar spine was done. It revealed: vertebral and spinal cord injury with an incarcerated $8 \mathrm{~cm}$ broken knife blade, complete spinal cord transection at T11 - T12 level, and hyper intense hematoma along the wound tract (Figure 1). The patient was operated as an emergency under general anesthesia with endotracheal intubation. A midline posterior approach extending between T9 and T12 levels was performed. Opening of the superficial dorsal aponeurosis immediately revealed the incarcerated knife blade. Para spinal muscles were severed subperiostly, spinous processes removed and T11 T12 laminectomy was done. It was noticed that the knife had broken the T12 spinous process, torn the spinal dura, transected the spinal cord and got stuck into the inferior part of T11 vertebral body. The incarcerated broken knife blade was gently pulled out with a rongeur. This revealed the spinal cord transection with a compressive hematoma at the injury site. The hematoma was removed with smooth suction and spinal cord wound washed with warm normal saline. Dura opening was sutured with 3/0 nylon sutures and the wound closed in layers (Figure 2). The immediate postoperative period was marked by a rapid recovery of motility and sensation so that at two weeks after surgery, muscle strength was at least 3 in all muscle groups ensuring motility of lower limbs till the legs. Nevertheless, motility of the feet remained null (level L5 and below). The patient was discharged from hospital on day 14 after surgery for a rehabilitation center. Thirty two months after surgery, patient's neurological status was unchanged although he mentioned a slight improvement in sphincter disturbances.

\section{Discussion}

There are not enough studies on the prevalence of traumatic spinal cord injuries (SCI) for a global estimation [6]. The average annual worldwide incidence of traumatic SCI is estimated at 35 cases per one million inhabitants with extremes of 10 and 83 cases per one million inhabitants per year. One third of SCI patients are tetraplegic and half have complete spinal cord transection. The mean age of these patients is 33 years and there is a net male predominance with 3.8/1 sex ratio [2] [6]. Nevertheless, spinal cord stab injuries (SCSI) remain rare and their frequency diversely estimated [1] [2] [4] [5] [7]. Their frequency was estimated at 26\% by Velmahos cited by Yeung [5] and 25\% by Peacock [8]. Most publications on spinal cord stab injury are based on case reports or limited case series [2]. Only two studies from South Africa concerned hundreds of cases, 450 cases in the series from Peacock [8] and 259 cases in the series of Lipschitz [9]. These high frequencies at that time could be explained by limited access to guns in that country which favored assaults by knives because there is not a recent study which corroborates the high frequencies of stab injuries found in those old series. In USA, out of 1736 cases 


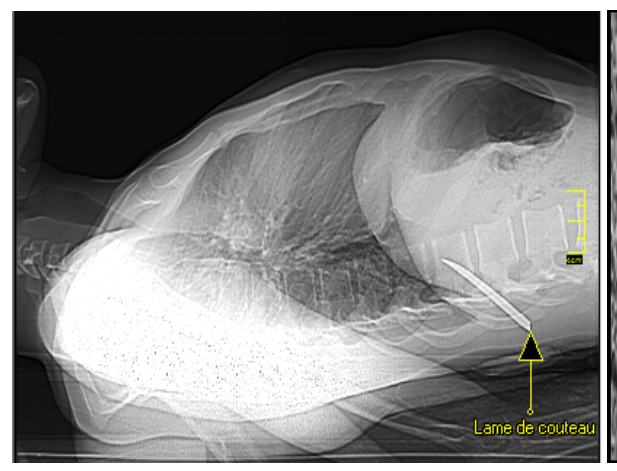

(a)

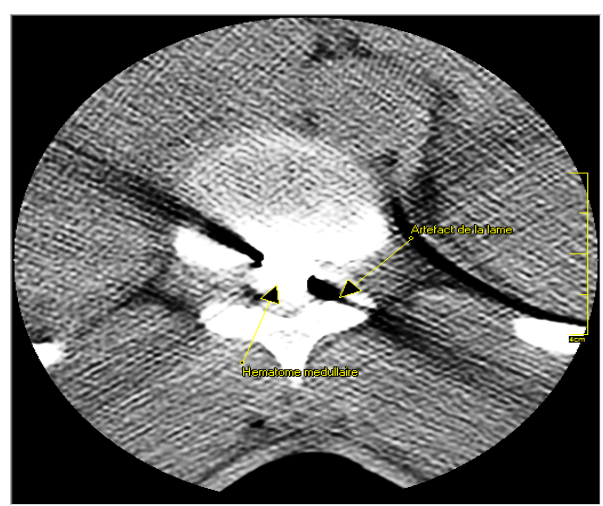

(c)

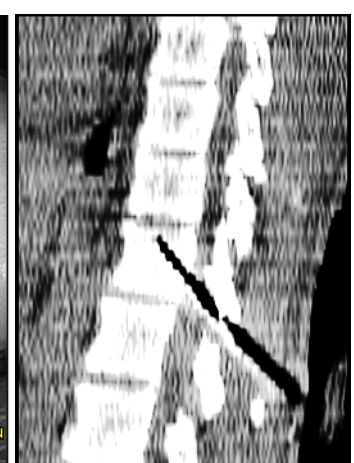

(b)

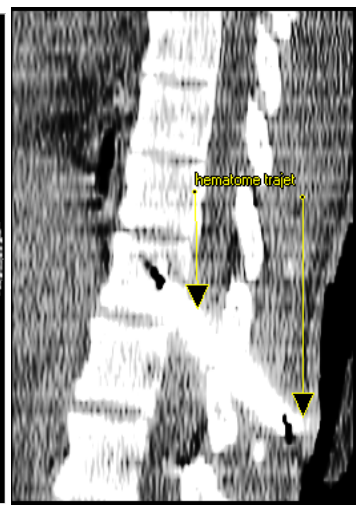

(d)

Figure 1. Spine computed tomography scans. (a) Scoot view showing the incarcerated broken knife blade (arrow); (b) Hypo intensity due to artifact from metallic foreign body (arrow); (c) Axial view showing blood clot within spinal cord (upward arrow) and hypo intense artifact from the knife blade (downward arrow); (d) Hyper intensity due to hematoma along the wound tract (arrows).

of traumatic SCI, 810 were caused by voluntary violence with 760 (43.77\%) caused by gunshots, and only 50 (2.88\%) caused by stabbing. Again, of the 16,024 cases of traumatic SCI recorded by the National Spinal Cord Injury Statistical Center (NSCISC), only 1.1\% resulted from stab injury [1]. In Cameroon, there is not a published study on the frequency of SCI due to gunshots or stabbing.

Spinal cord stab injuries (SCSI) are most often located in the thoracic spine as in this case, followed by cervical and lumbar areas [2] [3] [5] [8] [9]. The usual clinical presentation of SCSI is incomplete paraplegia manifesting most often as Brown-Séquard syndrome [1] [4] [8]. In the case reported here, paraplegia was complete. The majority of patients with SCSI have sphincter disturbances most often urinary. Complete spinal cord transection as in this case is rarer. Our observation has revealed that part of the neurologic symptoms can be provoked by hematoma within or around the spinal cord or post traumatic edema. Rapid recovery of muscle strength in most muscle groups in this case was probably due to release of spinal cord compression caused by intra spinal cord hematoma (Figure 2). The patient came with complete paraplegia at the thoracic level 10, but two weeks later, the persistent neurological deficits concerned only levels from L5 and below. This neurological status persisted until the last follow up visit (32 months). Other mechanisms which can explain acute neurological deficits in patients with SCI are: transection of nervous elements, spinal cord infarct due to blood vessels' injury or compression, and more rarely spinal cord epidural hematoma [4].

The radiological assessment of SCSI comprises: plain X-rays, computed tomography (CT) and magnetic resonance imaging (MRI). MRI is the gold standard in the absence of metal incarceration. It assesses the extent and evolution of SCI [3] [5]. CT scanning was informative enough in the reported case and allowed appropriate management of the patient.

As with any less frequent neurological pathology, the optimal management of SCSI is still yet to be determined. 


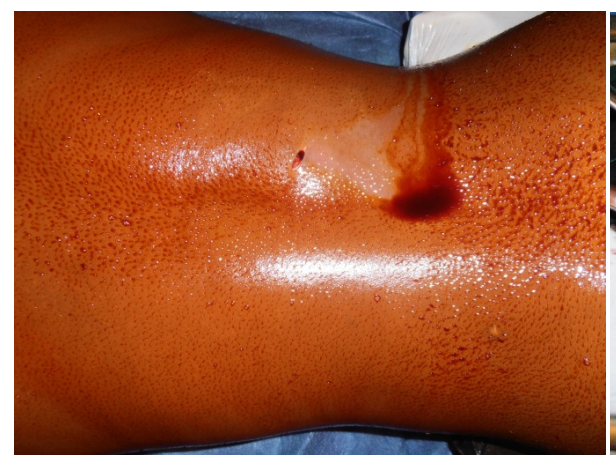

(a)

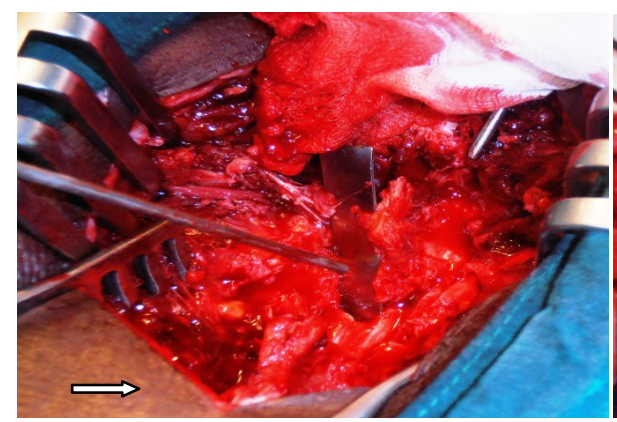

(c)

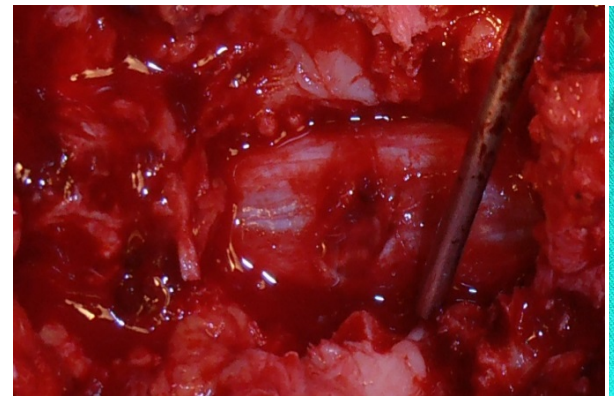

(e)

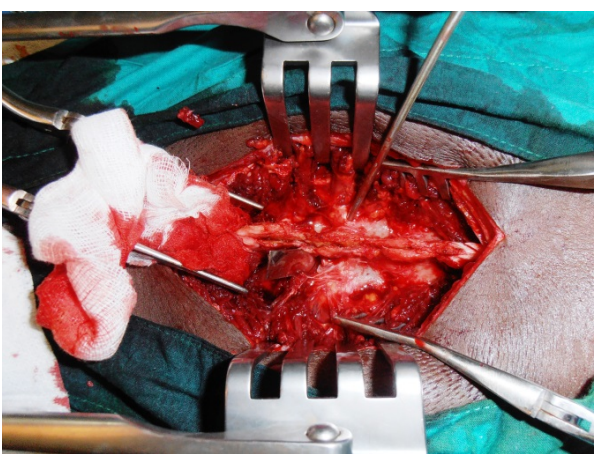

(b)

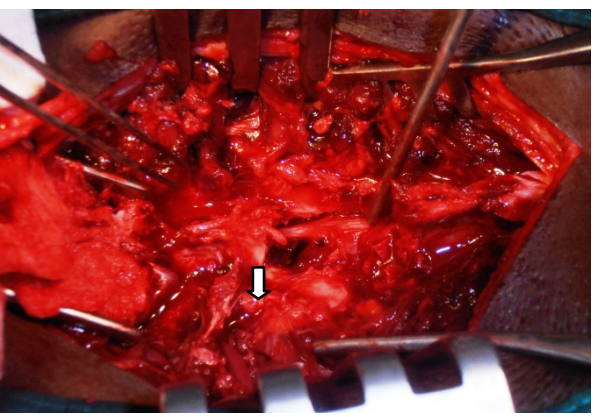

(d)

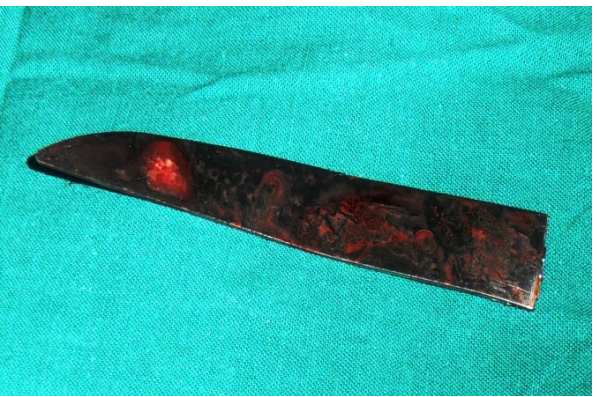

(f)

Figure 2. Intraoperative pictures. (a) Stab wound entry point with CSF leakage (arrow); (b) Incarcerated broken knife blade revealed after skin incision (arrow); (c) Spinal cord injury is shown after laminectomy was done (arrow); (d) Intramedullary hematoma shown after removal of knife blade (arrow); (e) Spinal cord aspect after removal of intramedullary blood clot; (f) The broken knife blade removed from the wound.

Injuries without incarceration of foreign bodies and without neurological deficits can be managed conservatively after assessment of spinal cord lesions by MRI [4]. Nevertheless, some authors do recommend surgery in such cases because surgery has shown benefit on associated lesions which are infection, cerebrospinal fluid (CSF) leakage and arachnoiditis [2] [3]. For Li et al. [2], surgery is crucial for neurological recovery no matter the initial neurological status. Surgery is indicated in case of incarceration of the knife blade because it allows safe removal under direct visual control and management of associated injuries (hematomas, CSF leak) which are source of complications and can cause secondary neurological deficits [2] [3]. Blind removal of foreign body is to be avoided because it can aggravate spinal cord injuries and provoke uncontrollable bleeding. The absence of benefit of surgery observed in SCI from gunshots with low velocity should not be extrapolated to SCSI as there is no study done in this direction.

Concerning prognosis, SCSI have a heavy impact on the victims, families and the entire society in terms of autonomy loss, working time loss, functional disability, the financial and human costs. Nevertheless, SCSI has a better prognosis (motor recovery and sphincter control) than other traumatic SCI, especially injuries due to guns. 
This is explained by the fact that in SCSI, incomplete injuries are much more frequent [1] [3] [4] [7]-[9]. More than half of incomplete paraplegics recover autonomy for walking with or without prosthesis and $71 \%$ in the series from Waters et al. achieved voluntary control of their bladder voidance [1]. Yeung has pointed out the role of spinous processes which make midline penetration of the knife more difficult [5]. Conversely, motor recovery with walk autonomy is very rare in case of complete neurological deficit as it was in the reported case. The T10 level complete paraplegia in our patient and it rapid regression till L5 level could be explained by release of cord compression due to associated hematoma (Figure 2).

\section{Conclusion}

Traumatic SCI have a heavy socio-economic impact. They constitute a heavy burden both for its social and medical aspects not only in the acute phase, but also during the first years because of complications (urinary infections, pressure sores, and behavioral disturbances) and the need for home care. For the case of spinal cord stab injuries in particular, victims are most often young men around thirty who were taking care of their families. They are suddenly handicapped, sometimes for life, thereby becoming a burden for the society and their relatives. Despite the fact that our young patient recovered useful muscle strength from the hips to the heels, because motor deficit remained null in his feet, he was unable to walk anymore and became a heavy burden for his family.

\section{Acknowledgements}

We thank mister D.S. for giving his consent for publication of his case. We thank Doctor NENG Humphry for helping with corrections.

We also thank MABO Anasthasie Pulcherie for her financial support.

\section{Conflict of Interest}

Authors declare no conflict of interest.

\section{References}

[1] Waters, R.L., Sie, I., Adkins, R.H. and Yakura, J.S. (1995) Motor Recovery Following Spinal Cord Injury Caused by Stab Wounds: A Multicenter Study. Paraplegia, 33, 98-101. http://dx.doi.org/10.1038/sc.1995.23

[2] Li, X., Curry, E.J., Blais, M., Ma, R. and Sungarian, A.S. (2012) Intraspinal Penetrating Stab Injury to the Middle Thoracic Spinal Cord with No Neurologic Deficit. Orthopedics, 35, 770-773. http://dx.doi.org/10.3928/01477447-20120426-40

[3] Saeidiborojeni, H.M., Moradinazar, M., Saiediborojeni, S. and Ahmadi, A. (2013) A Survey on Spinal Cord Injuries Resulting from Stabbings; A Case Series Study of 12 Years' Experience. Journal of Injury and Violence Research, 5, 70-74.

[4] Elgamal, E.A. (2004) Complete Recovery of Severe Quadriparesis Caused by Stab Wound at the Craniocervical Junction. Neurosurgical Review, 28, 70-72. http://dx.doi.org/10.1007/s10143-004-0359-0

[5] Yeung, J. and Karim, A. (2012) Complete Spinal Cord Transection from a Stab Wound with Surgical Precision. Journal of Emergencies, Trauma, and Shock, 5, 204. http://dx.doi.org/10.4103/0974-2700.96503

[6] Wyndaele, M. and Wyndaele, J.J. (2006) Incidence, Prevalence and Epidemiology of Spinal Cord Injury: What Learns a Worldwide Literature Survey? Spinal Cord, 44, 523-528. http://dx.doi.org/10.1038/sj.sc.3101893

[7] O’Neill, S., McKinstry, C.S. and Maguire, S.M. (2004) Unusual Stab Injury of the Spinal Cord. Spinal Cord, 42, 429-430. http://dx.doi.org/10.1038/sj.sc.3101602

[8] Peacock, W.S., Shrosbee, R.B. and Key, A.D. (1977) Reviews of 450 Stab Wounds of the Spinal Cord. South African Medical Journal, 51, 961-964.

[9] Lipschitz, R. (1976) Stab Wounds of the Spinal Cord. In: Vinjen, P.J. and Bruyn, G.W., Eds., Handbook of Clinical Neurology, North Holland Publishing, Amsterdam, 406-409. 